\title{
SINDROME DE DANDY-WALKER: A PROPÓSITO DE 4 CASOS
}

\author{
Gilberto Machado de Almeida *
}

Dá-se o nome de síndrome de Dandy-Walker a um tipo de hidrocéfalo no qual o IV ventrículo, muito dilatado, adquire o aspecto de um grande cisto (fig. 1). A condição tem sido exaustivamente estudada $3,5,9,10,17,27$, $31,32,35$, havendo relativa concordância quanto aos achados morfológicos e anátomo-patológicos: a fossa posterior, aumentada de volume à custa da posição anormalmente alta da tenda do cerebelo e do abaulamento do occipital, acha-se quase que totalmente preenchida pelo cisto correspondente ao IV ventrículo; o vérmis cerebelar, muito pequeno, apresenta-se rudimentar, estando ausente ou malformada a sua porção posterior; os hemisférios cerebelares, recalcados para a frente, também apresentam alterações em sua estrutura, principalmente disgenesia de núcleos cinzentos. A membrana do cisto, correspondente ao teto do IV ventrículo, na maioria das vêzes apresenta três camadas: a interna constituída pelo epêndima, às vêzes descontínuo; a externa, correspondente à pia-aracnóide; a intermediária, contendo células gliais, tecido colágeno (restos da tela corióidea) e, mais raramente, células de Purkinje ${ }^{17}$ ou fibras nervosas ${ }^{27}$. Geralmente os orifícios de Luschka e Magendie não são permeáveis; excepcionalmente um dos forames de Luschka permanece aberto ${ }^{12,17,31}$, o que tem ocasionado confusão na interpretação de alguns exames complementares, como a pneumencefalografia ou a prova dos corantes. Importante é a ausência de sinais inflamatórios.

Histórico - Há certa dificuldade para a classificação dos casos referidos na literatura antiga, tanto pela precariedade de alguns relatos como pela confusão com outras malformações cerebelares, em época em que a existência dos orifícios de Luschka e Magendie era discutida 2, 9, 10. Por isso as várias revisões são discordantes. Os casos referidos até 1914 são todos duvidosos, sendo citados em algumas revisões e excluídos de outras: Salter (1 caso, 1852 - cit. por Fowler e Alexander $\left.{ }^{12}\right)$, Hilton (1 caso, 1863 - cit. por Gibson ${ }^{17}$ e Russel ${ }^{27}$ ), Virchow (1 caso, 1863 - cit. por Gibson ${ }^{17}$ ), von Recklinghaussen ( 1 caso, 1864 - cit. por Gibson ${ }^{17}$ ), Fusari (1 caso, 1891 - cit. por Bertrand e col.4, Brodal e col.5, Gibson ${ }^{17}$, Sahs ${ }^{28}$, Shryock ${ }^{32}$ e Taggart e Walker ${ }^{35}$ ), Rossi (2 casos, 1891 e 1892 - cit. por Bertrand e

Trabalho da Clínica Neurológica da Fac. Med, da Univ. de São Paulo (Prof. Adherbal Tolosa).

* Neurocirurgião. 
col.4, Brodal e col. ${ }^{5}$, Gibson ${ }^{17}$, Shryock ${ }^{32}$ e Taggart e Walker ${ }^{35}$ ), Solovtzoff ( 2 casos, 1901 - cit. por Baker 1 e Sidenberg e col. ${ }^{35}$ ), Trevor e Rolleston ( 1 caso, 1911 - cit. por Brodal e col.5), Woskressenski (1 caso, 1911 - cit. por Brodal e col.5). Mesmo após 1914 existem casos duvidosos: Obersteiner (1 caso, 1916 - cit. por Bertrand e col. ${ }^{4}$, Sahs ${ }^{2: 3}$ e Taggart e Walker ${ }^{35}$ ) e Lyssenkow (1 caso, 1931 - cit. por Bertrand e col. ${ }^{4}$, Brodal e col. ${ }^{5}$, Sahs ${ }^{23}$ e Taggart e Walker ${ }^{35}$ ).

Em 1914 Dandy e Blackfan ${ }^{10}$ fizeram a primeira descrição pormenorizada. Dandy 9 em 1921 relatou mais 3 casos, aventando a hipótese de que a condição seria devida a falha de desenvolvimento dos orifícios que comunicam o IV ventrículo com o espaço subaracnóideo e não do seu fechamento como conseqüência de traumatismo ou infecção. Em 1942 Taggart e Walker ${ }^{35}$ reviram o assunto adotando a hipótese sugerida por Dandy. Em 1954, Benda $^{3}$ sugeriu o nome de "sindrome de Dandy-Walker" em substituição ao de "atresia do orifício de Magendie", usado até então.

Revendo a literatura a partir de 1914 encontramos 60 casos típicos: Dandy e Blackfan ${ }^{10}$ (1 caso, 1914), Dandy ${ }^{9}$ ( 3 casos, 1921), Pines e Surabaschwile (1 caso, 1932 - cit. por Bertrand e col.4, Brodal e col. ${ }^{5}$, Sahs ${ }^{28}$, Shryock ${ }^{32}$ e Taggart e Walker ${ }^{30}$ ), Castrillon (1 caso, 1933 - cit. por Bertrand e col. ${ }^{4}$, Brodal e col. ${ }^{5}$, Coleman e col. ${ }^{8}$, Sahs ${ }^{28}$, Shryock ${ }^{32}$ e Taggart e Walker ${ }^{35}$ ), Scarf ${ }^{30}$ (1 caso, 1933), Sahs ${ }^{28}$ (1 caso, 1941), Cohen (1 caso, 1942 - cit. por Brodal e col.5, Fowler ${ }^{12}$ e Taggart e Walker ${ }^{35}$ ), Taggart e Walker ${ }^{35}$ ( 3 casos, 1942), Shryock e Alexander ${ }^{32}$ (1 caso, 1943), Walker ( 1 caso, 1944 - cit. por Brodal e col. ${ }^{5}$, Coleman ${ }^{8}$, Fowler ${ }^{12}$ e Holland ${ }^{19}$ ), Sidenberg e Kessler ${ }^{33}$ (1 caso, 1946), Coleman e Troland 8 (2 casos, 1948), Russel 27 (1 caso, 1949), Schwarzkopf (1 caso, 1950 - cit. por Brodal e col.5), Van Epps ${ }^{36}$ (2 casos, 1953), Benda ${ }^{3}$ (6 casos, 1954), Schreiber e Reye ${ }^{31}$ (5 casos, 1954), Maloney 23 (2 casos, 1954), Gibson 17 (2 casos, 1955), Fowler e Alexander ${ }^{12}$ ( 3 casos, 1956), Matson ${ }^{25}$ ( 8 casos, 1956), Hemmer ${ }^{18}$ ( 7 casos, 1958), Clarke e Laidlow 7 (1 caso, 1958), Brodal e Hauglie-Hanssen 'j (2 casos, 1959), Holland e Graham ${ }^{19}$ (1 caso, 1959), Scarcella 29 (2 casos, 1960).

Também têm sido descritos alguns casos em animais: Lesbre e Forgeot (1 caso em bezerro, 1901 - cit. por Bertrand e col.4, Brodal e col. ${ }^{5}$ e Sahs ${ }^{28}$ ), Bertrand, Medynski e Salles ${ }^{4}$ (1 caso em cão, 1936), Dow ${ }^{11}$ (2 casos em cães, 1940), Verhaat ${ }^{37}$ (1 caso em cabra, 1942), Bonnevie e Brodal (uma raça de camundongos, 1946 - cit. por Brodal e Hauglie-Hanssen ${ }^{5}$ ).

Patogenia - Pràticamente todos os autores concordam em que a causa seja um defeito de desenvolvimento. Processos inflamatórios poderão determinar bloqueio dos orifícios de Luschka e Magendie; entretanto, em tais circunstâncias, faltam os outros elementos que caracterizam a síndrome. Dandy ${ }^{9}$ admitiu que a falha de desenvolvimento dos orifícios de Luschka e Magendie fôsse o defeito inicial, sendo esta teoria desenvolvida por Taggart e Walker ${ }^{35}$. Êstes orifícios devem estar permeáveis do $4 .^{\circ}$ ao $6 .^{\circ}$ mês de vida intra-uterina; se isto não se der, haverá hipertensão no sistema ven- 
tricular que se dilata. Este aumento é mais intenso ao nivel do IV ventrículo, determinando a separação das duas porções da comissura lateral e dificultando a diferenciação da parte posterior do vérmis cerebelar. $O$ lobo

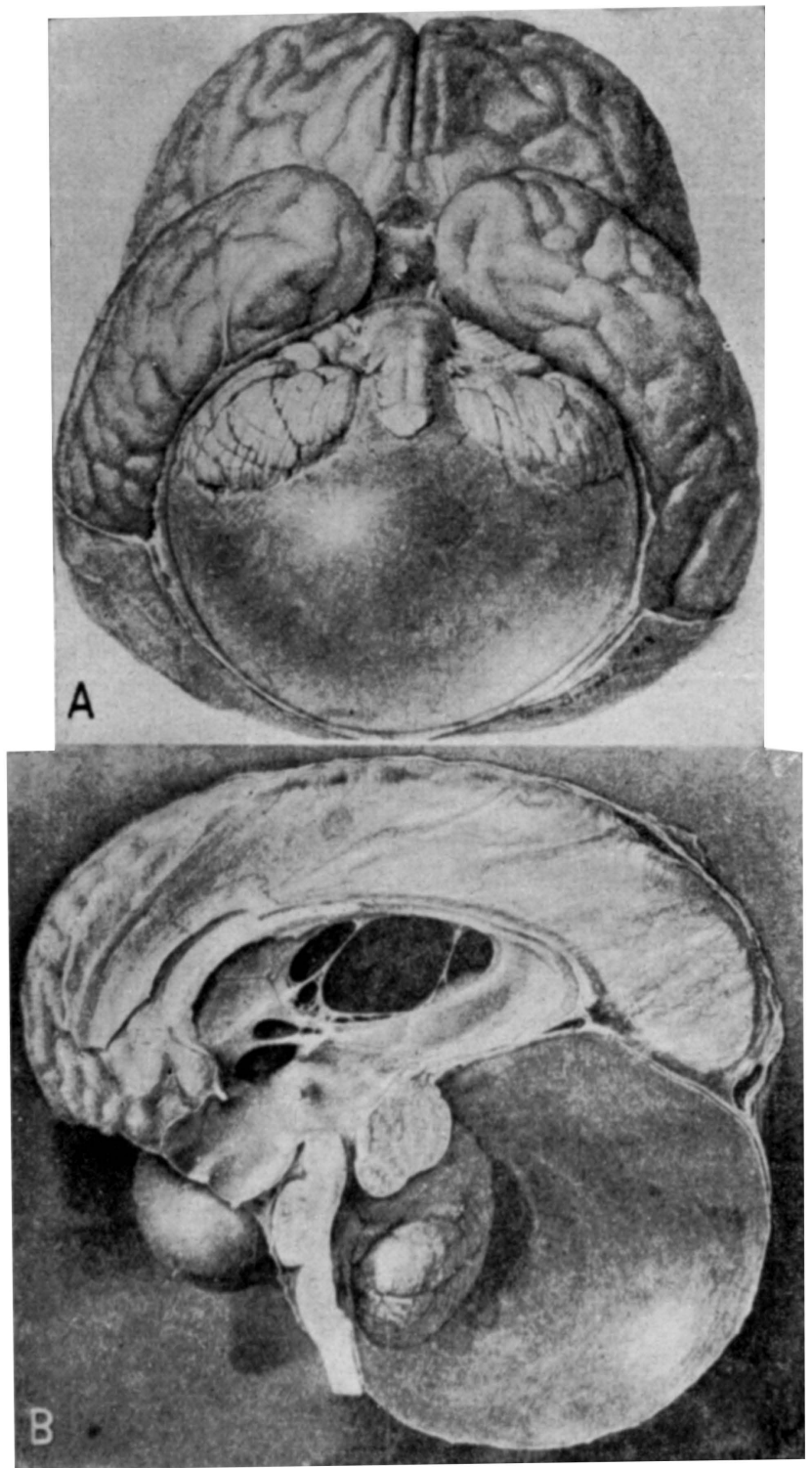

Fig. 1 - Desenho esquemático mostrando os principais aspectos encontrados na sindrome de Dandy-Walker (reproduzido do trabalho de Dandy ${ }^{9}$ ). 
anterior e os hemisférios cerebelares serão melhor formados porque neste período já estão diferenciados, tal não acontecendo com o vérmis posterior.

Benda ${ }^{3}$, em 1954, pretendeu demonstrar que a atresia do orifício de Magendie não é o fator essencial, que o quadro pertence à categoria dos defeitos de fechamento ou rachischisis e que a falha essencial está na ausência de fusão na linha média. Brodal e Hauglie-Hanssen ${ }^{5}$ também não aceitam a "atresia" dos orifícios do IV ventriculo como causa primária. Estudando uma raça de camundongos em que a moléstia se transmitia como gen mono-hibrido recessivo, êstes autores encontraram anomalias no cerebelo e no teto do IV ventrículo em estádio anterior à formação dos forames de Luschka e Magendie; estas anomalias mostravam que, já neste período, havia hipertensão cuja etiologia não foi esclarecida. Concluem êstes autores admitindo que a causa da sindrome existe antes do terceiro mês de vida intra-uterina e que a falha de abertura dos orifícios é apenas uma conseqüência. De fato, existem casos em que um ou dois orifícios são permeáveis 12,17,31; por outro lado, em cérebros normais tem sido assinalada a ausência de um ou dois forames ${ }^{3,9}$. Ültimamente Gardner 13, 14, 15, 16 voltou à teoria defendida por Taggart e Walker, mostrando que a síndrome de Dandy-Walker, a malformação de Arnold-Chiari, a siringomielia, a hidromielia, a meningomielocele, a encefalocele e, mesmo, alguns casos de hidrocéfalo comunicante são, meramente, expressões diferentes de um mesmo defeito, ou seja, falha de desenvolvimento normal dos orificios do teto do IV ventrículo. O estabelecimento de uma ou outra entidade, assim como das várias associações entre elas depende de fatôres diversos, principalmente da duração da alteração de permeabilidade do teto do IV ventrículo e da maior ou menor resistência oferecida pelas paredes do sistema ventricular ao aumento da pressão.

\section{CASUISTICA}

CaSo 1 - J. R., com 5 meses de idade, sexq masculino, branco, brasileiro, internado em 19-7-1957 (Reg. G. 479214). Desde o nascimento, disjunção acentuada das suturas cranianas. Aos 3 meses de idade os pais notaram aumento acentuado e progressivo do crānio. Algumas crises convulsivas generalizadas. Exame cliniconeurológico: crânio aumentado de volume, com certo abaulamento na regiáo occipital (perimetro craniano de $54 \mathrm{~cm}$ e torácico de $41,5 \mathrm{~cm}$ ), circulação venosa visivel, disjunção acentuada de suturas, fontanelas amplas e tensas. Movimentação diminuida. Reflexos superficiais e profundos normais; reflexo de Moro esboçado; reflexos de Magnus Klein ausentes. Marcha reflexa presente. Transiluminação (fig. 2A): transparência em tôda fossa posterior, que se apresenta aumentada, com elevação da tenda do cerebelo. Electrencefalograma (só foi obtido o registro do sono) : desaparecimento da atividade elétrica rápida em ambas as áreas occipitais; o exame sugere a existência de processo expansivo ou de coleção líquiida em ambas as áreas occipitais. Radiografias do crânio: aumento generalizado dos diâmetros cranianos; posiçāo anormal, nos parietais, dos sulcos produzidos pelos seios transversos. Sinugrafia direta (injeção de contraste no seio longitudinal superior): não há bloqueio; situaçāo anormalmente alta do torcular de Herófilo e dos seios transversos. Prova de corante (punção do ventriculo direito e injeção de $0,1 \mathrm{ml}$ de azul de toluidina a 5\%): o corante passou para o ventriculo esquerdo mas não para o espaço subaracnóideo lombar (30 minutos). O liquor obtido dos ventriculos tinha composição 

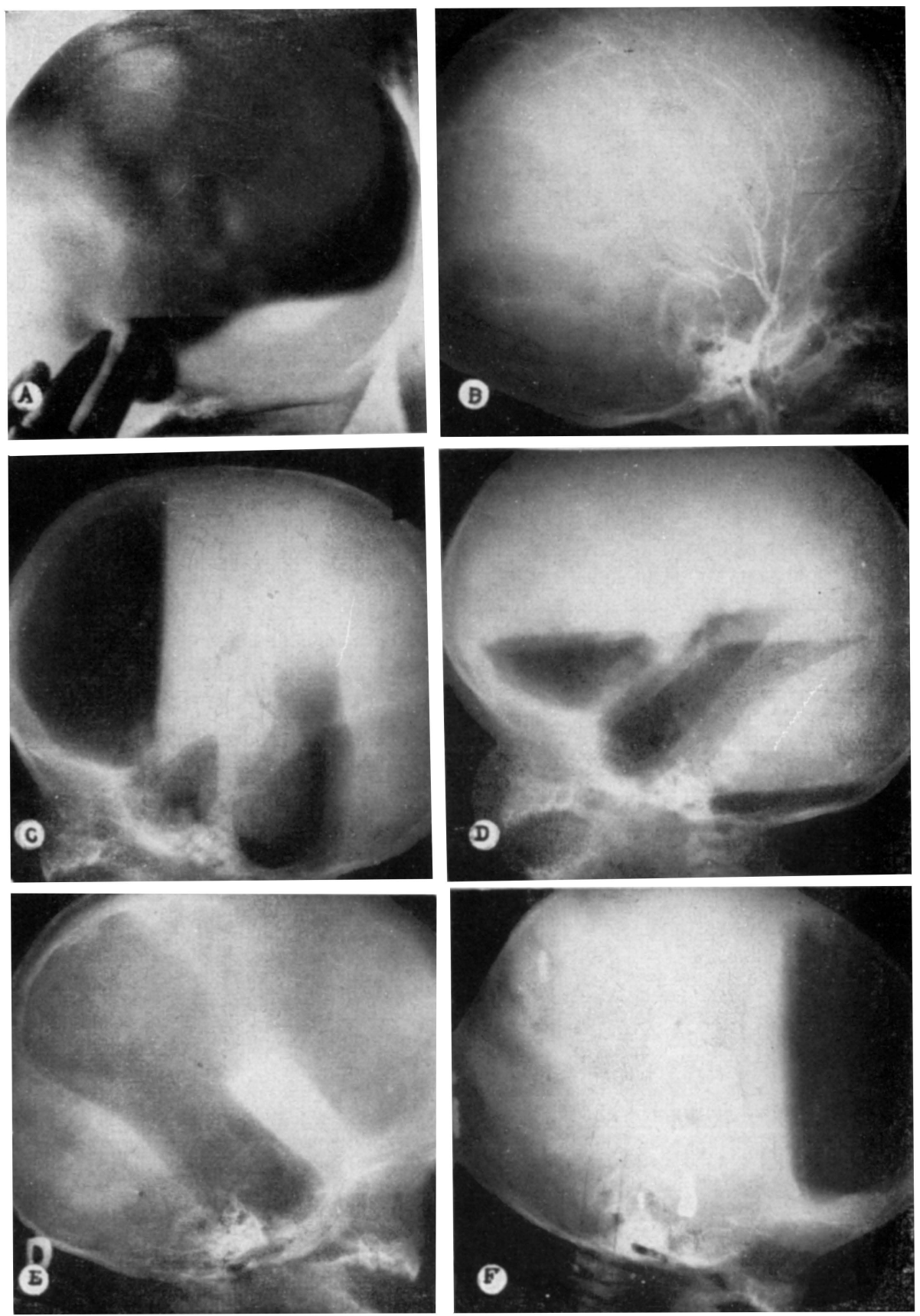

Fig. 2 - C'aso 1. Em A, transiluminação: transparência na região da fossa posterior. $\operatorname{Em} B$, pan-angiografia: elevação das artérias cerebrais médias e posteriores, ausência de vascularização na área correspondente ao cisto da fossa posterior. Em C, pneumoventriculogrfia: IV ventriculo transformado em grande cisto, aparecendo os limites do cerebelo atrófico. Em $D$, pneumoventriculografia, em incidência de perfil. com o paciente de cabeca para baixo, para mostrar o prolongamento cervical do cisto. Em $E$, pneumoventriculografia (incidencia de perfil, com o paciente deitado): elevação das porçōes posteriores dos ventriculos laterais e III ventriculo. $E m$, iodoventriculografia confirmando a posição do III ventrículo e o diverticulo cervical do IV ventricuio. 
normal mas no líquor lombar foi encontrada hipercitose de 56 células (linfomonócitos). Pan-angiografia cerebral: sinais indicativos de grande hidrocefalia interna; na incidencia lateral, elevação dos sistemas arteriais médio e posterior, sendo que êste último grupo apresenta curvatura de concavidade póstero-inferior, limitando grande área avascular correspondente ao cisto da fossa posterior (fig, 2B). Pneumoventriculografia (13-8-57) e pneumencefalografia mais pneumocistografia (17-8-57): hidrocéfalo bloqueado por obstrução ao nivel da transição ventrículo-subaracnóidea; a fossa posterior, grandemente aumentada pela elevação da tenda do cerebelo, apresenta-se quase totalmente preenchida por enorme cisto correspondente ao IV ventriculo (fig. 2C e D); êste clsto apresenta uma expansão para o canal raqueano até $C_{1}-C_{2}$ visivel na chapa de perfil com o paciente de cabeça para baixo (fig. 2D); cerebelo reduzido a pequena formação na porção anterior da fossa posterior (fig. 'C); grande dilatação dos ventriculos laterais com elevação dos cornos occipitais; tercelro ventrículo dilatado e com elevação da sua porção posterior (fig. 2E); o trânsito entre os III e IV ventriculos foi feito com dificuldade. Pela punção lombar só foi possivel retirar $30 \mathrm{ml}$ de liquor. $O$ ar injetado por essa via contrastou o espaço subaracnóideo cervical, a cisterna magna reduzida de volume, as cisternas pré-pontina, interpenducular, ambiens e silviana; não houve contrastação da cisterna optoquiasmática e do espaço subaracnóideo periencefálico. Iodoventriculografia (fig. 2F) : o contraste passou lentamente do III ao IV ventriculo através de um aqueduto bastante estreito e algumas gôtas foram ter à expansão cervical do cisto.

Operação - Foi resolvido abrir apenas a expansāo cervical do cisto, através de laminectomia de $\mathrm{C}_{1}$ e $\mathrm{C}_{2}$ e alargamento do buraco occipital. Após abertura da dura mater e da aracnóide foi vista a expansão referida e retirado um fragmento da membrana, determinando saida abundante de liqüido claro. Exame histológico da membrana: lâmina de tecido fibroso (pia-aracnóide), revestido por elementos de derivação ependimária. No pós-operatório a fontanela ficou deprimida mas surgiu hipertonia em extensão dos quatro membros e hipertermia. A criança apresentou pjora das condições gerais, vindo a falecer com broncopneumonia, 36 dias após o ato cirúrgico.

Necropsia - Broncopneumonia. Craniectomia de fossa posterior e laminectomia cervical. Grande dilatação do sistema ventricular. Elevação da tenda do cerebelo. $O$ ventriculo rombencefálico apresenta-se transformado em grande cisto que comprime os hemisférios cerebelares para frente e para os lados. Vermis rudimentar e elevado. O aspecto encontrado é pràticamente idêntico ao esquema apresentado por Dandy ${ }^{3}$ (fig. 1), havendo apenas um orificio na membrana, conseqüência do ato cirúrgico. Não havia reação inflamatória nas meninges.

CaSo 2 - A. S. S., com 2 meses de idade, sexo masculino, branco, brasileiro, internado em 16-4-1959 (Reg. G. 553201). Desde o nascimento a criança apresentava crânio maior do que o normal; uma semana antes da internação passou a alimentar-se mal e a gemer muito. Exame fisico - Crânio muito aumentado, medindo $64 \mathrm{~cm}$, com rêde venosa bem desenhada, de consistência mole, ossos muito separados. Sinal do sol poente. Distrofia. Exame neurológico igual ao de criança recémnascida. Transiluminação: positiva em todo o crânio. Electrencefalograma: ausência da atividade elétrica rápida nas áreas occípito-parietais. O caso foi considerado sem possibilidades terapêuticas devido ao grande volume do crânio e ao sofrimento cerebral grave. Alguns dias após a internação a criança apresentou sinais de broncopneumonia, vindo a falecer em 28-4-59.

Necropsia - Bronquite e bronquiolite purulenta. Grande hidrocefalia, córtex cerebral muito delgado. $O$ aspecto encontrado na fossa posterior era semelhante ao esquema apresentado por Dandy ${ }^{9}$ (fig. 1 ).

Caso 3 - L. C., com 6 meses de idade, sexo feminino, branca, brasileira, internada em 24-3-1959 (Reg. G. 544898). Desde o nascimento apresentava a cabeça 
alongada e uma tumoração na região posterior; o crânio aumentou progressivamente de volume mas a meningocele permaneceu inalterada. Exame fisico - Perimetro craniano de $51 \mathrm{~cm}$; meningocele occipital com $6 \mathrm{~cm}$ de diâmetro (fig. 3A). Teste de Gesell com 24 semanas: desenvolvimento normal. Transiluminação: positiva através da meningocele e negativa no crânio. Electrencefalograma normal. Radiografia do crânio: considerável aumento de todos os diâmetros; fontanelas e suturas alargadas; crânio bifido occipital com meningocele; não foram evidenciados os sulcos dos seios transversos.

A paciente permaneceu cêrca de 60 dias usando Acetozolamida (40 $\mathrm{mg}$ por $\mathrm{kg}$ ao dia) sem sucesso, pois o perímetro do crânio aumento para $53,5 \mathrm{~cm}$. Novo teste de Gesell, feito 2 meses e meio após o primeiro, mostrou pequeno progresso neste periodo, ficando entāo bem demonstrado o retardo de desenvolvimento. Prova do corante (punção do ventrículo esquerdo e injeção de $0,5 \mathrm{ml}$ de fenolsulfonftaleina): o corante passou para o ventrículo direito, mas não para o subaracnóideo lombar. O líquor obtido dos ventrículos não apresentava anormalidade, mas no liquüido lomjar havia hipercitose de 12 células (linfomonócitos).

Em 2-6-59, foi feita derivação ventrículo-jugular segundo a técnica de Spitz e Holter. No dia seguinte a criança teve amigdalite aguda e, depois, broncopneumonia, falecendo em 4-6-1951. No periodo pós-operatório a derivação permaneceu drenando bem, pois a fontanela ficou flácida e ligeiramente deprimida.

Necropsia - Amigdalite purulenta. Abscessos pulmonares múltiplos. A válvula e as sondas colocadas no ato cirúrgico estavam em boa posição, não apresentando obstrução. Meningocele occipital. Ventrículo rombencefálico com os aspectos típi$\cos$ da sindrome de Dandy-Walker. A meningocele não continha expansão do IV ventrículo.

CASo 4 - O. H., com 5 anos de idade, sexo masculino, branco, brasileiro, internado em 10-12-1959 (Reg. G. 568391). O menino nasceu de parto de nádegas, demorado; com um mês de idade os pais notaram aumento acentuado do volume craniano; a partir dos 8 meses o crescimento do crânio tornou-se menos acentuado; a criança sustentou a cabeça com 1 ano, sentou-se com 2 anos e começou a falar e a andar apoiado aos 2 anos e meio; aos 14 meses apresentou crise convulsiva demorada no hemicorpo esquerdo e, depois, hemiparesia do mesmo lado; com ano e meio começou a ter crises de hipertonia dos 4 membros e opistótono, durante as quais vomitava; após estas crises, que se repetiam cada 15-20 dias, a criança ficava abtida e sonolenta; nos meses que precederam a internação acusou dôres na nuca. Exame clínico-neurológico - Crânio aumentado de volume, principalmente à custa de sua porção posterior; perímetro craniano de $60 \mathrm{~cm}$ (perímetro torácico $=55 \mathrm{~cm}$ ); fontanelas e suturas fechadas. Discreta hipertonia em extensão dos membros inferiores. Déficit motor generalizado, maior à esquerda. Reflexos profundos vivos nos membros inferiores, especialmente à esquerda. Sinal de Babinski bilateral. Ligeira palidez das papilas ópticas. Transiluminação: negativa. Teste de Gesell: retardo nitido principalmente no setor da motricidade. Electrencefalografia normal. Radiografias do crânio: além dos sinais típicos de hidrocefalia, chama a atenção a posição anormalmente alta (acima da sutura lambdóldea) da impressão dos seios transversos (fig. 3B). Radiografias da coluna vertebral: espina bifida oculta de $\mathrm{S}_{1}$. Sinugrafia direta (via seio longitudinal superior, após perfuraçāo frontal): situação alta do confluente e dos seios transversos (fig. 3C). Pneumoventriculografia: dilatação de tôdas as cavidades ventriculares; elevação das porções posteriores dos ventriculos laterais; o IV ventriculo acha-se transformado em um cisto que ocupa quase tôda a fossa infra-tentorial, apresentando ainda uma expansão que ultrapassa o plano do forame occipital chegando até $\mathrm{C}_{2}$ (fig. 3D).

Com êsses dados foi feito o diagnóstico de síndrome de Dandy-Walker e o paciente foi submetido à derivação ventrículo-jugular segundo a técnica de SpitzHolter, em 5-1-1960. Pós-operatório ótimo; o paciente não apresentou mais cefaléia ou vômitos e continua a fazer os exercícios de recuperação. 

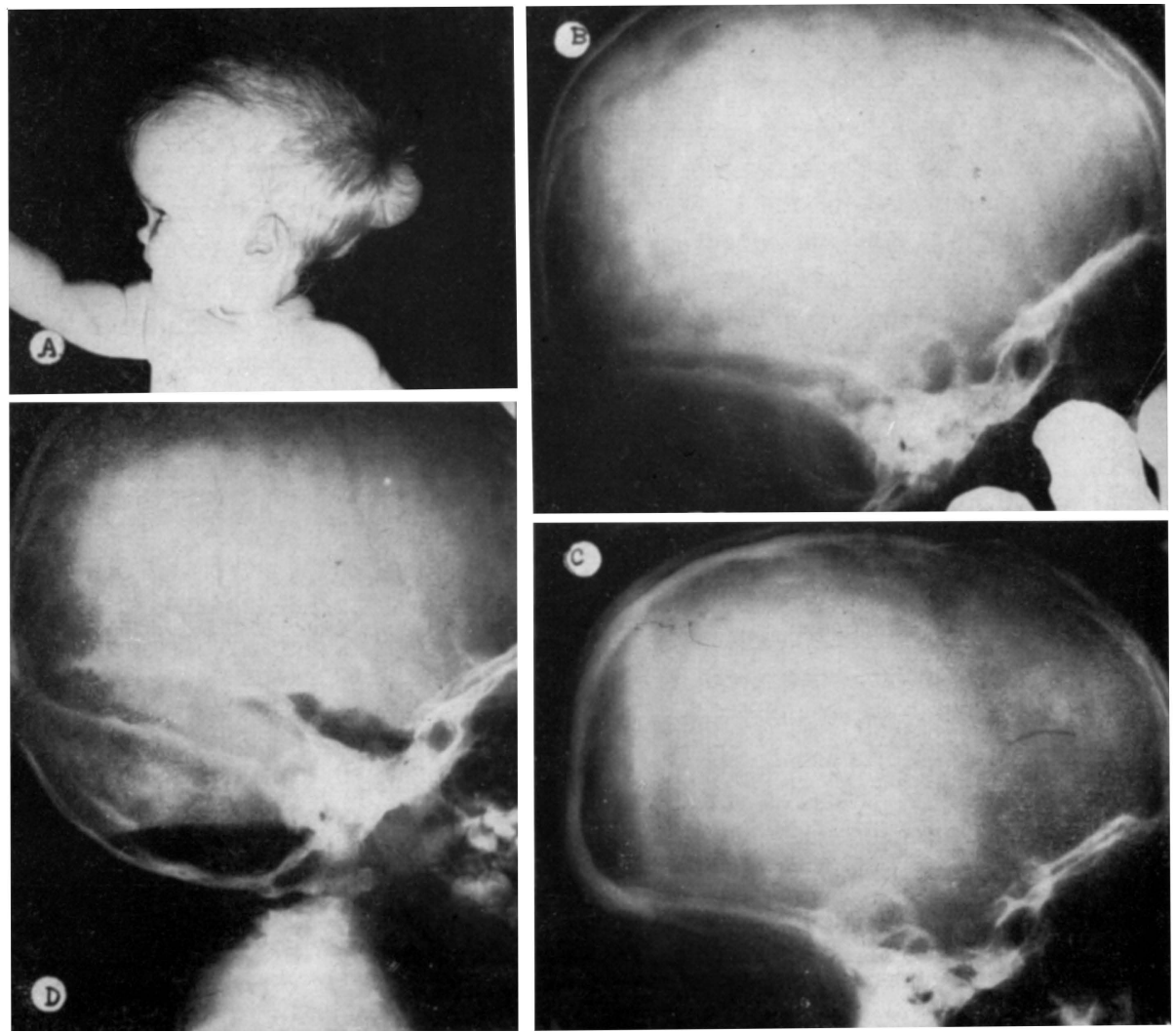

Fig. 8 - Em A (caso 3), fotografia mostrando a meningocelc $e$ a forma alongada do crânio. $\operatorname{Em} B$ (caso 4), craniograma: visibilização dos sulcos dos seios transversos nos parietais. Em C (caso 4), sinugrafia confirmando a situaço dos seios transversos. Em $D$ (caso 4), pneumoventriculografia: incidência de perfil, com o paciente de cabeça para baixo, mostrando o grande cisto da fossa posterior $e$ o prolongamento cervical.

\section{COMENTARIOS}

Geralmente as manifestações da doença surgem desde o nascimento ou nos primeiros meses de vida. O quadro se confunde com os demais tipos de hidrocéfalo; entretanto, pela inspeção, pode ser levantada a suspeita diagnóstica quando o crânio seja alongado (fig. 3A), ou quando exista abaulamento ao nível da fossa posterior. Pela palpação pode-se notar um afastamento relativamente grande dos bordos da sutura lambdóide.

Outras vêzes o paciente, com ou sem retardo psíquico-motor, vive bem até certa idade, quando então surgem sinais de hipertensão intracraniana ou convulsões. Traumatismos relativamente pequenos podem desencadear ou 
exacerbar a sindrome de hipertensão 8, 17, 18, 31. Admite-se que o traumatismo rompa o equilíbrio instável que vinha sendo mantido por vários fatôres: diminuição da produção de líquor, permeabilidade de um dos orifícios de Luschka, diálise através da membrana e reabsorção do líquor pelo epêndima ou por vasos da própria parede do cisto. Punções lombares ${ }^{28}$ ou ventriculares 9 também podem acarretar desequilíbrio, determinando exacerbação da sintomatologia. Nos pacientes em que a hipertensão intracraniana aumenta sùbitamente podem ocorrer rigidez de nuca ${ }^{28}$, opistótono ${ }^{5,33}$ ou crises de hipertonia ${ }^{5}$. O nosso caso 4 apresentou freqüentes crises de hipertonia e opistótono possivelmente devidas à compressão do tronco cerebral determinada por acentuação da hipertensão intracraniana.

Em alguns casos registrados na literatura o diagnóstico só foi feito mediante necropsia de pacientes que faleceram por outras causas; em alguns dêsses casos, durante a vida, fôra assinalada síndrome cerebelar (caso de Shryock e Alexander ${ }^{32}$ ) ou deficit mental (casos de Castrillon cit. por Sahs ${ }^{28}$ e de Pines e Surabaschwile cit. por Bertrand e col. ${ }^{4}$, Brodal e col. ${ }^{5}$, Shryock ${ }^{32}$ e Taggart e Walker ${ }^{35}$ ). Síndrome cerebelar uni ou bilateral tem sido encontrada com certa freqüência $5,8,18,23,28,32$ 33. Em nossos casos 1 , 3 e 4 ocorreram convulsões, o que também tem sido referido ${ }^{3,17}$. O caso 3 apresentava meningocele occipital (fig. 3A) semelhante à encontrada em um dos pacientes de Schreiber e Reye ${ }^{31}$. Já foi assinalada a associação com outras malformações, dentro e fora do estojo crânio-raquídeo $31,32,33,35,36$, principalmente com agenesia do corpo caloso $31,33,36$.

Transiluminação - Fowler e Alexander ${ }^{12}$ descrevem, como típico, o fato de a luz atravessar a fossa posterior, não o fazendo no restante do crânio; a área transiluminada é limitada pela linha de implantação da tenda do cerebelo. Em nossos pacientes, êste fato foi observado uma única vez (caso 1, fig. 2A). No caso 3 , a transiluminação foi positiva em todo o crânio devido à grande dilatação do sistema ventricular. Nos demais havia opacidade total, pois se tratava de crianças maiores, com hidrocéfalo menor.

Electrencefalograma - Não têm sido referidas, na literatura, alterações electrencefalográficas características. O exame foi feito em todos os nossos casos. Nos dois primeiros (casos 1 e 2), em que o cisto da fossa posterior era muito grande, notou-se o desaparecimento do ritmo rápido nas áreas posteriores. Êste dado pode constituir um elemento a mais para o diagnóstico da síndrome de Dandy-Walker.

Craniograma - Além dos sinais radiográficos conseqüentes à hipertensão intracraniana, podemos encontrar elementos que permitem levantar a suspeita ou mesmo firmar o diagnóstico de síndrome de Dandy-Walker. Taggart e Walker ${ }^{35}$ foram os primeiros a chamar a atenção para a posição anormalmente alta, nos ossos parietais e não nos occipitais, dos sulcos dos seios transversos e do confluente; isto é causado pelo aumento do conteúdo da fossa posterior, impedindo a migração normal dêstes seios venosos. Este 
sinal, considerado patognomônico, pode, entretanto, não ser encontrado, ou por não estarem os seios venosos em posição anormal 3,17, 19 ou devido a não visibilização dos sulcos ${ }^{27,31}$, o que constitui a regra em crianças pequenas. Outros dados radiológicos de menor valor são: dolicocefalia, abaulamento da fossa posterior, disjunção relativamente grande da sutura lambdóide e adelgaçamento do occipital. O exame radiográfico foi feito em 3 de nossos casos: em um (caso 3) o sulco dos seios transversos não era visível; nos dois outros (casos 1 e 4) pôde ser demonstrada a posição anormal dêstes seios venosos. Os outros sinais radiológicos são menos evidentes e, considerados isoladamente, não permitem firmar o diagnóstico.

Sinugrafia direta - A importância dêste exame foi ressaltada por Ingraham e Matson 20, Matson ${ }^{25}$ e Fowler ${ }^{12}$; êle permite verificar a posição dos seios transversos, tendo especial valor quando os sulcos correspondentes não aparecem de maneira nítida no craniograma. O sinugrama, realizado em dois dos nossos casos (1 e 4), confirmou a posição anormal da tórcula e dos seios transversos. Este sinal, quando presente, pode ser considerado patognomônico.

Arteriografia - Este exame não tem sido empregado para o diagnóstico da sindrome de Dandy-Walker. Entretanto, no caso 1 a pan-angiografia cerebral mostrou sinais interessantes: em incidência lateral a região da fossa posterior apresentava-se avascularizada e limitada, superiormente, pelas artérias cerebrais posteriores, que se encontravam elevadas e descreviam curva de concavidade para baixo; a posição destas artérias confirmou a situação alta da tenda do cerebelo.

Líqüido cefalorraquidiano - O exame de líquor e a injeção de corante no sistema ventricular têm sido feitos na maioria dos casos de crianças pequenas, com fontanela aberta, demonstrando a existência de bloqueio entre os sistemas ventricular e subaracnóideo lombar. Isto, entretanto, não constitui regra absoluta, pois, em alguns casos ${ }^{3},{ }^{31}$ há passagem do corante de um compartimento para outro, através de um orifício de Luschka aberto ou por diálise ao nivel da membrana. Em pacientes de maior idade, tem sido feito o exame do líquor colhido por via lombar sem que tenham sido obtidos dados de interêsse. Nos casos 1 e 3 a prova dos corantes mostrou bloqueio; além disso, no líquor obtido por via lombar havia discreta hipercitose. Nos outros pacientes o exame não foi realizado.

Pneumoventriculografia - Êste exame, mostrando dilatação maior ou menor do sistema ventricular, com características especiais, é o que mais freqüentemente tem confirmado o diagnóstico: nos ventrículos laterais há elevação dos cornos occipitais e das porções posteriores dos cornos temporais; o IV ventrículo é substituído por um grande cisto, que oclipa quase tôda a fossa posterior e que se projeta para a coluna cervical, chegando a atingir a segunda vértebra cervical 12,25. Esta projeção cervical pode ser observada em chapas laterais com o paciente de cabeça para baixo e é con- 
siderada, por Matson ${ }^{25}$, como indício patognomônico. Quando o exame é feito com bastante contraste pode ser delimitado o cerebelo atrofiado (fig. 2C). Maloney ${ }^{23}$ chama a atenção para os casos em que há necessidade de fazer perfurações, pois podem ser atingidos os seios venosos como aconteceu em um de seus pacientes; Schreiber e Reye ${ }^{31}$ relataram um caso em que as perfuraçōes atingiram o cisto. Realizamos o exame em dois pacientes: no caso 4 os achados foram típicos e confirmaram o diagnóstico; no caso 1 chamou-nos a atenção uma dificuldade de trânsito ao nível do aqueduto, confirmada pela iodoventriculografia e provàvelmente ocasionada por hérnia do conteúdo da fossa posterior, através da incisura da tenda, determinando compressão do mesencéfalo ${ }^{6}$. Em um dos casos de Taggart e Walker ${ }^{35}$ não houve passagem de ar do III para o IV ventriculo, provàvelmente pela mesma causa. No trabalho de Sahs ${ }^{28}$ existe uma fotografia em que a herniação descrita é nitida.

Pneumencefalografia - Na maioria das vêzes êste exame não fornece dados de interêsse, podendo mesmo levar à confusão com casos de hidrocefalia comunicante, devido à passagem de ar para o sistema ventricular $3,7,31$. Isto se deve à permeabilidade de pelo menos um dos orifícios de Luschka, pois admite-se que o ar não possa atravessar a membrana do cisto ${ }^{25}$, como acontece com os corantes. Êstes dados mostram a precariedade da prova dos corantes e da pneumencefalografia no diagnóstico da síndrome de DandyWalker. Entretanto, êstes exames podem ter valor no estudo das condições do espaço subaracnóideo, o que tem interêsse na conduta terapêutica ${ }^{9,21,22}$, pois, havendo bloqueio neste compartimento, a simples retirada da membrana correspondente ao teto do IV ventrículo transformará a hidrocefalia de bloqueada em comunicante ${ }^{25}$. No caso 1 a pneumencefalografia mostrou permeabilidade apenas parcial do sistema subaracnóideo, o que, entretanto, permitiu, no curto período pós-operatório, a reabsorção do líquor, pois a fontanela permaneceu deprimida.

Iodoventriculografia - Êste exame não tem sido citado na literatura. Uma única vez o realizamos e o resultado foi sem dúvida interessante, pois, além de comprovar a existência da projeção do cisto para a região cervical, permitiu um estudo mais detalhado do III ventrículo e do aqueduto de Sylvius que tinham sido mal delimitados pelas pneumografias; a porção posterior do III ventrículo achava-se muito elevada, dando ao todo um trajeto quase vertical e o aqueduto, geralmente descrito como dilatado, encontrava-se estreitado, explicando a dificuldade na passagem do ar do III para o IV ventrículo.

Terapêtica - Dos 60 casos citados na literatura, 36 foram submetidos à cirurgia: em 33 foi feita craniectomia de fossa posterior com retirada de porção maior ou menor da parede do cisto. Os resultados obtidos em 3 pacientes não foram relatados; dos 30 restantes, $13(43,3 \%)$ faleceram (um após nova tentativa por meio de derivação ventrículo-subaracnóidea), 4 $(\mathbf{1 3 , 3} \%)$ foram ulteriormente submetidos à derivação subaracnóideo-ureteral ${ }^{25}$, em um caso $(3,3 \%)$ permaneceu a hipertensão intracraniana, em 12 
(40\%), o resultado foi considerado bom. As demais operações tentadas: ventriculostomia do III ventrículo ${ }^{35}$, electrocoagulação dos plexos coróides ${ }^{3}$ e derivação subaracnóideo-ureteral ${ }^{31}$ tiveram maus resultados.

Em nosso primeiro paciente foi feita retirada parcial da parede do cisto (porção cervical), o que determinou descompressão brusca e sofrimento do tronco do encéfalo, ocorrendo o óbito no $36^{\circ}$ dia de pós-operatório. Os casos 3 e 4 foram submetidos à derivação ventrículo-jugular segundo a técnica de Spitz, utilizando a válvula de Holter; o sistema drenou o líquor satisfatòriamente nos dois casos, mas um dos pacientes veio a falecer devido a processo infeccioso.

A remoção da parede do cisto tem dado resultados relativamente precários. Esta operação, além de mais traumatizante, tem o inconveniente de necessitar de um sistema subaracnóideo livre, sem o que há transformação da hidrocefalia de bloqueada em comunicante, exigindo nova intervenção ${ }^{25}$. O bloqueio do espaço subaracnóideo pode ser ocasionado por vários fatôres: a) falha de desenvolvimento do espaço, provocada pelo deficiente trabalho de dissecção normalmente realizado pelo líquor; b) compressão do espaço subaracnóideo periencefálico pela hipertensão no interior dos ventrículos; c) hernição de conteúdo da fossa posterior através da incisura da tenda ${ }^{6}$. Êstes fatos explicam grande parte dos fracassos cirúrgicos, principalmente em crianças. Nos pacientes mais idosos o sistema subaracnóideo geralmente se encontra em melhores condições, sendo mais animadores os resultados obtidos pela cirurgia. Outro inconveniente da retirada da membrana é a possibilidade de descompressão brusca, como ocorreu com o nosso primeiro caso. Êstes inconvenientes não existem com a derivação ventrículojugular que é pouco traumatizante, não depende das condições do espaço subaracnóideo e não determina descompressão brusca. Infelizmente no caso 3 o resultado foi mau devido a uma intercorrência. A operação de SpitzHolter não foi citada na literatura sôbre a sindrome de Dandy-Walker e a nossa casuística é pequena; entretanto, a experiência adquirida em outros tipos de hidrocefalia $24,26,34$ permite afirmar que esta técnica é superior à remoção da parede do cisto.

\section{CONCLUSOES}

1. O diagnóstico de síndrome de Dandy-Walker pode ser suspeitado ao exame clínico.

2. Na maioria dos casos o craniograma, mostrando os sulcos dos seios transversos nos parietais, ou a transiluminação, com aspecto típico, permitem afirmar o diagnóstico.

3. Nos casos em que as radiografias simples não mostram os sulcos dos seios transversos é necessário recorrer à sinugrafia.

4. A pneumoventriculografia foi o exame mais empregado. Atualmente, porém, pode ser dispensada, desde que o diagnóstico tenha sido firmado por 
exames menos traumatizantes. Em alguns casos, quando não há elevação nítida da tenda do cerebelo, o pneumoventriculograma ainda é o melhor método diagnóstico.

5. A prova dos corantes e a pneumencefalografia podem levar à confusão com formas de "hidrocefalia comunicante"; êstes exames têm interêsse apenas para o estudo das condições do espaço subaracnóideo.

6. O electrencefalograma às vêzes pode ser o primeiro elemento a levantar a suspeita diagnóstica.

7. Arteriografia e iodoventriculografia têm interêsse apenas teórico, complementando dados fornecidos pelos demais exames.

8. A derivação ventriculo-jugular (técnica de Spitz-Holter) é a melhor terapêutica para êstes casos, pois tem reais vantagens sôbre a técnica mais empregada até agora, ou seja a retirada da parede do cisto.

\section{RESUMO}

Dá-se o nome de síndrome de Dandy-Walker a um tipo de hidrocefalia cuja principal característica é a transformação do IV ventrículo em um grande cisto, que ocupa quase tôda a fossa posterior; esta, por sua vez, está aumentada, devido à elevação da tendá do cerebelo e abaulamento do occipital. A patogenia do processo é discutida; admite-se geralmente que se trate de um vício de desenvolvimento determinado pela falta de abertura dos orifícios de Luschka e Magendie.

Em revisão bibliográfica foram encontrados 60 casos. São apresentados mais 4, dois dos quais foram submetidos à derivação ventrículo-jugular pela técnica de Spitz-Holter. São discutidos os principais sinais clínicos, os exames subsidiários e a terapêutica. A posição anormalmente alta dos seios transversos, observada no craniograma ou na sinugrafia, é considerada como sinal patognomônico. A transiluminação do crânio também pode apresentar aspecto característico. Estes exames, quando tipicos, permitem evitar a realização de exames mais traumatizantes, como as ventriculografias. A terapêutica mais empregada tem sido a abertura da parede do cisto. Não há na literatura referência à operação de Spitz que, em nossa opinião, é a melhor forma de tratamento.

\section{SUMMARY}

The Dandy-Walker syndrome: considerations on four cases.

Dandy-Walker syndrome is a particular form of hydrocephalus whose main feature is the transformation of the IV ventricle into a large cyst, which occupies almost the whole of the posterior fossa; the latter, owing to the lifting of the tentorium cerebelli and bulging of the occipital bone, 
is enlarged. The pathogenesis is incertain; it is generally agreed that it is originated from a developmental disorder brought about by the non-opening of the foramina of Luschka and Magendie.

In the literature were found 60 cases reported. Four additional cases are reported, two of which underwent the ventriculo-jugular shunt by SpitzHolter's technique. The main clinical signs, laboratory data and therapy are discussed. The abnormally high position of the transverse sinuses, as seen in the X-ray of the skull or in the sinugram, is considered a pathognomonical sign. The trans-illumination of the skull may also show a characteristic aspect. When the results of these exames are typical, it is possible to avoid more traumatizing ones such as ventriculographies. The therapy more commonly used is the opening of the wall of the cyst. There is no reference in the literature to Spitz's operation, which is, in our opinion, the best treatment.

\section{REFERENCIAS}

1. BAKER, R. C.; GRAVES, G. O. - Cerebellar agenesis. A. M. A. Arch. Neurol, a. Psychiat., 25:548-555, 1931. 2. BARR, M. L. - Observations on the foramen of Magendie in a series of human brains. Brain, $71: 281-289,1948$. 3. BENDA, C. E. - The Dandy-Walker syndrome of the so-called atresia of the foramen Magendie. J. Neuropath. a. Exper. Neurol., 13:14-29, 1954. 4. BERTRAND, I.; MEDYNSKI, C.; SALLES, P. - Ettude d'un cas d'agénesie du vermis cérébelleux chez le chien. Rev. Neurol., 66:716-733, 1936. 5. BRODAL, A.; HAUGLIE-HANSSEN, E. - Congenital hydrocephalus with defective development of the cerebellar vermis (Dandy-Walker syndrome). J. Neurol., Neurosurg. a. Psychiat., 22:99-108, 1959. 6. CARREA, R.; GIRADO, M.; EURNEKIAN, A. - Hernia transtentorial de cerebelo, bloqueo tentorial y hidrocefalia. Actas y Trabajos del VI Congreso Latinoamericano de Neurocirurgia, Montevideo, págs. 103-111, 1955. 7. CLARKE, E.; LAIDLOW, J. - Silent hydrocephalus. Neurology, 8:382-386, 1958. 8. COLEMAN, C. C.; TROLAND, C. E. - Congenital atresia of the foramina of Luschka and Magendie with report of 2 cases of surgical cure. J. Neurosurg., 5:84-88, 1948. 9. DANDY, W. E. - The diagnosis and treatment of hydrocephalus due to occlusions of the foramina of Magendie and Luschka. Surg., Gynec. a. Obst., 32:112-124, 1921. 10. DANDY, W. E.; BLACKFAN, K. D. - Internal hydrocephalus: an experimental, clinical and pathological study. Am. J. Dis. Child., 8:406-482, 1914. 11. DOW, R. S. - Partial agenesis of the cerebellum in dogs. J. Comp. Neurol., 72:569-586, 1940. 12. FOWLER, F. D.; ALEXANDER Jr., E. - Atresia of the foramina of Luschka and Magendie. A cause of obstructive hydrocephalus. A. M. A. J. Dis. Children, 92:131-137, 1956. 13. GARDNER, W. J. - Anatomic anomalies common to myelomeningocele of infancy and syringomyelia of adulthood suggest a common origin. Cleveland Clin. Quart., 26:118133, 1959. 14. GARDNER, W. J. - Anatomic features common to the Arnold-Chiari and the Dandy-Walker malformations suggest a common origin. Cleveland Clin. Quart., 26:206-222, 1959. 15. GARDNER, W. J.; ABDULlaH, A. F.; McCORMACK, L. J. - The varying expressions of the embryonal atresia of the fourth ventricle in adults: Arnold-Chiari malformation, Dandy-Walker syndrome, "arachnoid" cyst of the cerebellum and syringomyelia. J. Neurosurg., 14:561-607, 1957. 16. GARDNER, W. J.; GOODALL, R. J. - The surgical treatment of Arnold-Chiari malformation in adults: an explanation of jts mechanism and importance of encephalography. J. Neurosurg., 7:199-206, 1950. 17. GIBSON, J. B. - Congenital hydrocephalus due to atresia of the foramen of Magendie. J. Neuropath. a. Exper. Neurol., 14:244-262, 1955. 18. HEMMER, R. - Zum Hydrocephalus occlusus infolge congenitaler Missbildung am Ausgang des 4. Ventrikels. Arch. f. Psychiat. u. Zeitschr. f. d. ges. 
Neurol., 197:206-214, 1958. 19. HOLLAND, H. C.; GRAHAM, W. I. - Congenital atresia of the foramina of Luschka and Magendie with hydrocephalus: report of a case in an adult. J. Neurosurg., 15:688-694, 1959. 20. INGRAHAM, F. D.; MATSON, D. D. - Neuro-Surgery of Infancy and Childhood. Charles Thomas, Springfield (Illinois) 1954, págs. 154-156. 21. LAURENCE, K. M. - The urinary phenolsulphonphthalein (Phenol red) excretion test in hydrocephalus. Arch. Dis. Childhood, 32: 413-416, 1957. 22. LAURENCE, K. M. - Some applications of the urinary phenolsulphonphthalein excretion test in hydrocephalus and related conditions. Brain, 82: 551-565, 1959. 23. MALONEY, A. F. J. - Two cases of congenital atresia of the foramina of Magendie and Luschka. J. Neurol. Neurosurg. a. Psychiat., 17:134-138, 1954. 24. MATERA, R. F. - Tratamiento quirúrgico de la hidrocefalia en el niño (operación de Spitz-Holter). Rev. Brasil. Neurocir., 1:7-25, 1960. 25. MATSON, D. D. - Pre-natal obstruction of the fourth ventricle. Am. J. Roentgenol., 76:499-506, 1956. 26. McNAB, G. M. - The Spitz-Holter valve. J. Neurol. Neurosurg. a. Psychiat., 22:82, 1959. 27. RUSSEL, D. S. - Observations on the pathology of hydrocephalus. Med. Res. Council, Spec. Rep. Series, no 265, H. M. Stationary Office, Londres, 1949. 28. SAHS, A. L. - Congenital anomaly of the cerebellar vermis. Arch. Pathol., 32:52-63, 1941. 29. SCARCELLA, G. - Radiologic aspects of the Dandy-Walker syndrome. Neurology, 10:260-266, 1960. 30. SCARFF, J. E. - Spastic hemiplegia produced by a congenital cyst replacing the cerebellar vermis. J. Nerv. a. Ment. Dis., 78:400-401, 1933. 31. SCHREIBER, M. S.; REYE, R. D. - Posterior fossa cysts due to congenital atresia of the foramina of Luschka and Magendie. Med. J. Australia, 41:743-748, 1954. 32. SHRYOCK, E. H.; ALEXANDER, H. B. Congenital malformations of the cerebellar vermis associated with dilatations of the fourth ventricle and cisternal arachnoidal cyst: report of a case. Bull. Los Angeles Neurol. Soc., 8:11-17, 1943. 33. SIDENBERG, S. S.; KESSLER, M. M.; WOLPAW, R. - A case of tetralogy of Fallot with absence of cerebellar vermis; termination by brain abscess. J. Pediat., 28:719-728, 1946. 34. SPITZ, E. B. Neurosurgery in the prevention of exogenous mental retardation. Pediatric Clin. North America, 6:1215-1235, 1959. 35. TAGGART, J. K.; WALKER, A. E. - Congenital atresia of the foramens of Luschka and Magendie. A. M. A. Arch. Neurol. a. Psychiat., 48:583-609, 1942. 36. Van EPPS, E. F. - Agenesis of the corpus callosum with concomitant malformations, including atresia of the foramina of Luschka and Magendie. Am. J. Roentgenol,, 70:47-60, 1953. 37. VERHAART, W. J. C. Partial agenesis of the cerebellum and medulla and total agenesis of the corpus callosum in a goat. J. Comp. Neurol., 77:49-60, 1942.

Clinica Neurológica - Hospital das Clinicas da Fac. Med. da Univ. de São Paulo - Caixa Postal $\$ 461$ - São Paulo, Brasil. 